\title{
Experiencia con el blog colectivo de Sedic
}

Por Virginia Becerril, María Jesús Butera, Nuria Escudero, Julio Igualador, Elisa Prieto y Luis Rodríguez Yunta

\begin{abstract}
Resumen: Se analiza la experiencia llevada a cabo dentro de la asociación profesional Sedic para desarrollar un blog colaborativo como herramienta de comunicación capaz de crear una auténtica "conversación permanente" a través de la Red.

Palabras clave: Blogs colectivos, Biblioteconomía y documentación, Comunicación social, Política de calidad, Usabilidad.

\section{Title: An experience with Sedic's col-} laborative blog

Abstract: Description of the work carried out
by Sedic, a Spanish library association, for deve-
loping a collaborative blog as a communication
tool to enable authentic, ongoing conversations
over the Net.

Keywords: Collaborative blogs, Library and information science, Communication, Quality policies, Usability.
\end{abstract}

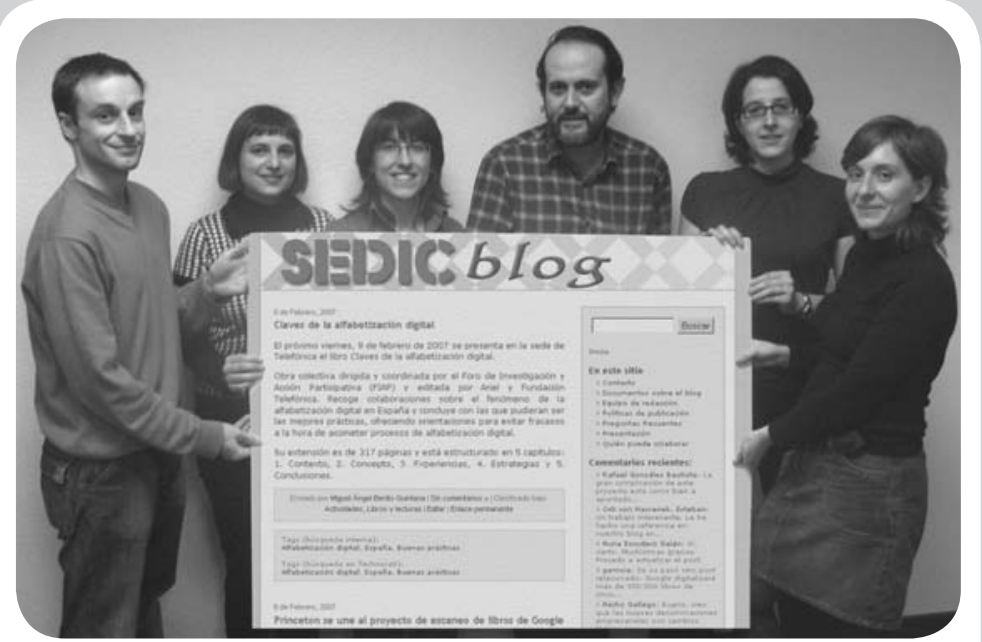

Julio Igualador, blogmaster; Virginia Becerril, documentalista en Over MCW (Marketing \& Comunication Wordwide) y lic. en Documentación por la Univ. de Salamanca; Elisa Prieto, Biblioteca y Centro de Documentación, Elzaburu SA; Luis Rodríguez Yunta, documentalista del Cindoc (CSIC), miembro de los grupos NormaWeb y Activa de Sedic; María Jesús Butera, documentalista de HayGroup, doctoranda en la Univ. Carlos III de Madrid; Nuria Escudero, documentalista en el Centro de Información del Medicamento (CIM) de Pfizer.

Becerril, Virginia; Butera, María-Jesús; Escudero, Nuria; Igualador, Julio; Prieto, Elisa; Rodríguez-Yunta, Luis. "Experiencia con el blog colectivo de Sedic". En: El profesional de la información, 2007, marzo-abril, v. 16, n. 2, pp. 134-137.

DOI: $10.3145 /$ epi.2007.mar.06

\section{Nacimiento y definición del proyecto}

\section{EL INTERÉS POR EL FOR-}

MATO BLOG ha estado presente por parte de algunos miembros activos de Sedic al menos desde el año 2003, con la idea de que la asociación pudiera utilizarlo para dar voz a sus grupos de trabajo o desarrollar un agregador de canales o hilos RSS que proporcionara a sus socios una especie de servicio de alerta de información de tipo profesional que fuera adoptando estándares de sindicación.

Sin embargo esos proyectos no llegaron a materializarse, de modo que se puede decir que el concepto de un blog colaborativo ligado a la idea de comunicación no meramente unidireccional toma forma a comienzos de 2006. El blog quedó definido en las primeras re- uniones de trabajo como "un lugar de encuentro y participación para los profesionales de la información (bibliotecarios, documentalistas y archiveros)".

\section{http://blog.sedic.es}

Los objetivos quedaron establecidos en los siguientes términos:

- Instaurar un canal de comunicación entre Sedic y sus socios así como las personas interesadas en sus actividades, a fin de poder recibir de forma pública la retroalimentación necesaria en forma de sugerencias y propuestas.

- Contribuir a un mayor impacto social de las actividades de la organización y de sus grupos de trabajo, de forma que se publiciten cursos, seminarios o iniciativas, pero además se puedan reflejar opiniones y comentarios sobre los resultados de las mismas.
- Disponer de un foro de difusión y debate para temas de interés profesional: noticias, novedades, imagen de la profesión, formación permanente, etc.

A partir de esta formulación de intenciones se llevó a cabo una primera difusión entre los socios a fin de sondear la posible acogida y captar personas interesadas en contribuir activamente. Esta pre-difusión coincidió además en el tiempo (primeros meses de 2006) con la impartición en la sede de Sedic del curso Blogs para profesionales de la información de Javier Leiva Aguilera, algunos de cuyos alumnos se animaron a sumarse al proyecto. De resultas de estas dos acciones pudo constituirse un grupo de socios cuyo interés y compromiso ha ido más allá de todo lo esperable y que conforman el núcleo de lo que es hoy el equipo de redacción.

http://blog.sedic.es/?page_id=19 


\section{Definición del modelo de comunicación y publicación}

Ya en las primeras reuniones de trabajo se entendió que la mejor manera de vehicular estos objetivos sería la implantación de un blog colaborativo, cuyos contenidos fuesen fruto de la libre cooperación entre socios, sin que esto excluyera las aportaciones de todos los profesionales de la información con independencia de su afiliación a Sedic.

Suele decirse que "un blog es una conversación", y tiene bastante de certero (esta bidireccionalidad de la comunicación es una de las causas del éxito y su popularización), pero también es cierto que en esta conversación no todos los interlocutores son iguales; el usuario/autor que publica entradas, artículos o posts tiene privilegios con respecto al usuario/lector cuya única opción suele ser la de añadir comentarios a esas entradas. A nuestro entender, es importante comprender esta distinción cuando se plantea la posibilidad o la conveniencia de un blog cooperativo como canal de comunicación institución / organismo / asociación <---> socios / miembros / colaboradores <---> interesados / usuarios / público.

Esta disparidad entre los actores de la comunicación puede dar la impresión de ser inconveniente o poco equitativa; existen modelos de intercambio de comunicación y publicación colaborativa en la Web que pueden parecernos distribuidos de un modo más igualitario (foros de debate, listas de distribución, wikis, etc.). Lo cierto es que no hay una solución ideal y unívoca y el mejor formato será el que mejor se adapte a la definición y objetivos que se quieren alcanzar. Sí entra dentro de nuestros requisitos el que la conversación haya de mantenerse ordenada y centrada, ceñida a ámbitos temáticos determinados, que haya de cumplir en todo caso unos mínimos en cuanto a calidad e interés, que requiera de cierta uniformidad en cuanto a estructura y presentación que nos permita asegurar a nuestros usuarios un mínimo de usabilidad, navegabilidad y legibilidad. Es probable que un modelo de publicación cooperativa completamente abierto (cualquier persona puede intervenir), paritario o parigual (todos los que intervienen poseen los mismos privilegios en cuanto a publicación) y libre (sin sistema alguno de moderación) no sea la mejor de las opciones y dé lugar a una conversación caótica y ruidosa (en el sentido de ruido informativo o documental). De hecho, no abundan los ejemplos de canales de comunicación que reúnan en puridad esta triple condición (abiertos, paritarios y sin moderación) tampoco entre foros de debate, listas de distribución, wikis, etc., cuando es conveniente evitar la dispersión temática y formal.

Nuestro reto, según hemos ido comprobando a lo largo de nuestra aún breve singladura, continúa siendo aunar en lo posible esta exigencia de calidad en los contenidos y en su organización y presentación con la consecución de una verdadera conversación: el fomento de la participación, la recepción de feedback de nuestros interlocutores, la asignación adecuada, el reparto de papeles a los intervinientes de manera que perciban que tienen voz dentro de esta conversación de un modo satisfactorio, la flexibilidad y comodidad del sistema de participación. Creemos que, de modo general, este reto es el mismo en cualquier canal de comunicación y publicación de información web que se pretenda colaborativo y no quiera convertirse en un mero órgano de transmisión de información unidireccional de carácter corporativo.

Finalmente el reparto de papeles quedó establecido tal y como se recoge en la sección "Quién puede colaborar":

http://blog.sedic.es/?page_id=9
- Redactores: dispuestos a colaborar de manera habitual, a adoptar y unificar criterios, a responsabilizarse del control de calidad de los contenidos y a desempeñar tareas de moderación y administración en el CMS (content management system).

- Colaboradores: con posibilidad de enviar sus contribuciones al sistema con la frecuencia que deseen.

- Usuarios: con capacidad para enviar comentarios a los artículos publicados.

Otro requisito que se estableció para seleccionar un sistema de gestión de contenidos es que permitiera establecer categorías temáticas. Aunque el procedimiento de navegación habitual en los blogs se basa en los archivos cronológicos, en un modelo colectivo donde se espera tratar gran variedad de temas por un número amplio de redactores y colaboradores resulta indispensable ofrecer al lector la opción de navegación por temas de interés.

Se establecieron apartados específicos para los grupos de trabajo de Sedic y otros de carácter más genérico (actividades, formación, libros y lecturas, nuestra profesión, nuestro blog, recursos, Sedic y visto y oído). Se redactó una pequeña definición o nota de alcance para cada categoría.

\section{Selección de la aplicación de gestión (CMS) de blogs}

Definido ya a grandes rasgos nuestro proyecto en cuanto a misión, objetivos y modelo de comunicación/publicación, comprobamos que necesitábamos una herramienta de gestión de contenidos que reuniera al menos las siguientes funciones:

- Posibilidad de asignar permisos o roles adecuados a cada uno de los actores implicados en este proceso de creación de contenidos, comunicación y publicación. 
- Dominio y hosting propios, que nos permitieran garantizar la nointrusión de publicidad indeseada, responsabilizarnos nosotros mismos de la disponibilidad en la web de nuestro blog sin caídas inesperadas, de la integridad de los datos mediante backups, etc. En definitiva, una opción que disminuyera en lo posible la dependencia de terceros no deseados que pudieran afectar negativamente a su credibilidad o funcionamiento.

- Recuperación de entradas a través de categorías preasignadas.

- Asimismo, era deseable la adecuación en la medida de lo posible a la arquitectura de la web de Sedic. Pronto se vio que este último punto no iba a resultar sencillo, pues la mayoría de los gestores de contenidos para blogs (y los mejores y más implantados entre ellos) se han desarrollado en la comunidad de software libre y requieren usualmente una arquitectura web basada en PHP/MySQL/Apache que el hosting y el desarrollo actual de la web de Sedic no reúne. Finalmente se optó por asumir el pequeño costo de la herramienta en un hosting diferente, propio, que cumpliera estos requisitos. Esta opción nos permitía emplear el $C M S$ más idóneo para nuestras necesidades, con más posibilidades de parametrización, de asignación y gestión de privilegios dentro del sistema, consolidado nacional e internacionalmente y una comunidad activa que, hoy por hoy, garantiza la actualización y la implementación de nuevos desarrollos en forma de plugins que es posible añadir al sistema.

La aplicación seleccionada fue WordPress. Una vez instalada se pudo abrir un período de parametrización en cuanto a funciones y organización de los contenidos, así como de definición y puesta en marcha de un diseño visual acorde (aunque específico) con el estilo visual corporativo de Sedic, tras lo cual el blog, ya funcional, entró en fase de pruebas de publicación.

http://www.wordpress.org

\section{Establecimiento de bases para estandarizar procedimientos y formatos}

Al tratarse de un proyecto de autoría colectiva, con la pretensión de tener el número más amplio posible de redactores y colaboradores, preveíamos una tendencia natural a la dispersión de contenidos, temática, tono e intención de los artículos, redacción, etc., así como ideas diversas en lo relativo a organización de los mismos. Era necesario proporcionar a los autores unas indicaciones comunes al respecto, por lo que se inició la redacción de un manual que marcase las pautas básicas de actuación. Se ha contado desde su inicio con una sección de documentos que pueden resultar de utilidad para cualquier profesional embarcado en un proyecto de creación de contenidos en la web. Un ejemplo de ello es el libro de estilo, cuya versión 1.0 se puso en línea el 1 de junio de 2006. En él se recogen los siguientes aspectos:

http://blog.sedic.es/docs/Libro_de_ estilo_v_1_0.pdf

- Definión de la política de contenidos del blog: líneas temáticas, derechos de autor, criterios de moderación y requisitos para aceptar una colaboración.

- Peticiones de claridad en la transmisión de información: uso de títulos significativos, aportación de datos completos sobre los actos que se citen.

- Recomendaciones de buenas prácticas: enlaces a fuentes.

- Normas de redacción: corrección gramatical y ortográfica, sintaxis, párrafos bien estructurados y tipografía.

- Aspectos iconográficos: uso de símbolos gráficos e imágenes.
"El carácter colaborativo

de la experiencia del blog

ha permitido elaborar

documentos que pueden

ser de utilidad para el conjunto de la blogosfera"

Con este material consensuado y elaborado, que nos permitiría mantener una cierta integridad en cuanto a presentación, temática y estructuración, se realizó una convocatoria pública a los socios para presentarlo en sociedad el 15 de junio de 2006. Esta "ceremonia de bautismo" pretendía así mismo animar a la participación. Para esta sesión se elaboró un folleto que resume informaciones sobre qué es un blog, cómo participar o cómo suscribirse a él a través de un agregador de contenidos RSS. Estábamos en marcha.

http://blog.sedic.es/?p=44

http://blog.sedic.es/docs/Blog_ SEDIC_folleto.pdf

\section{Algunos datos estadísticos} 2006:

Datos de junio a noviembre de

- Número de entradas o artículos: 121

- Comentarios publicados: 107

- Redactores y colaboradores: 15

- Enlaces entrantes: 82 (datos de Technorati a 4 de diciembre de 2006).

- Páginas visitadas: 34.423

- Visitantes: 16.230

\section{Conclusiones}

- Es importante destacar la documentación que se ha generado sobre normas de estilo y recomendaciones sobre buenas prácticas en la edición de contenidos en la web. 


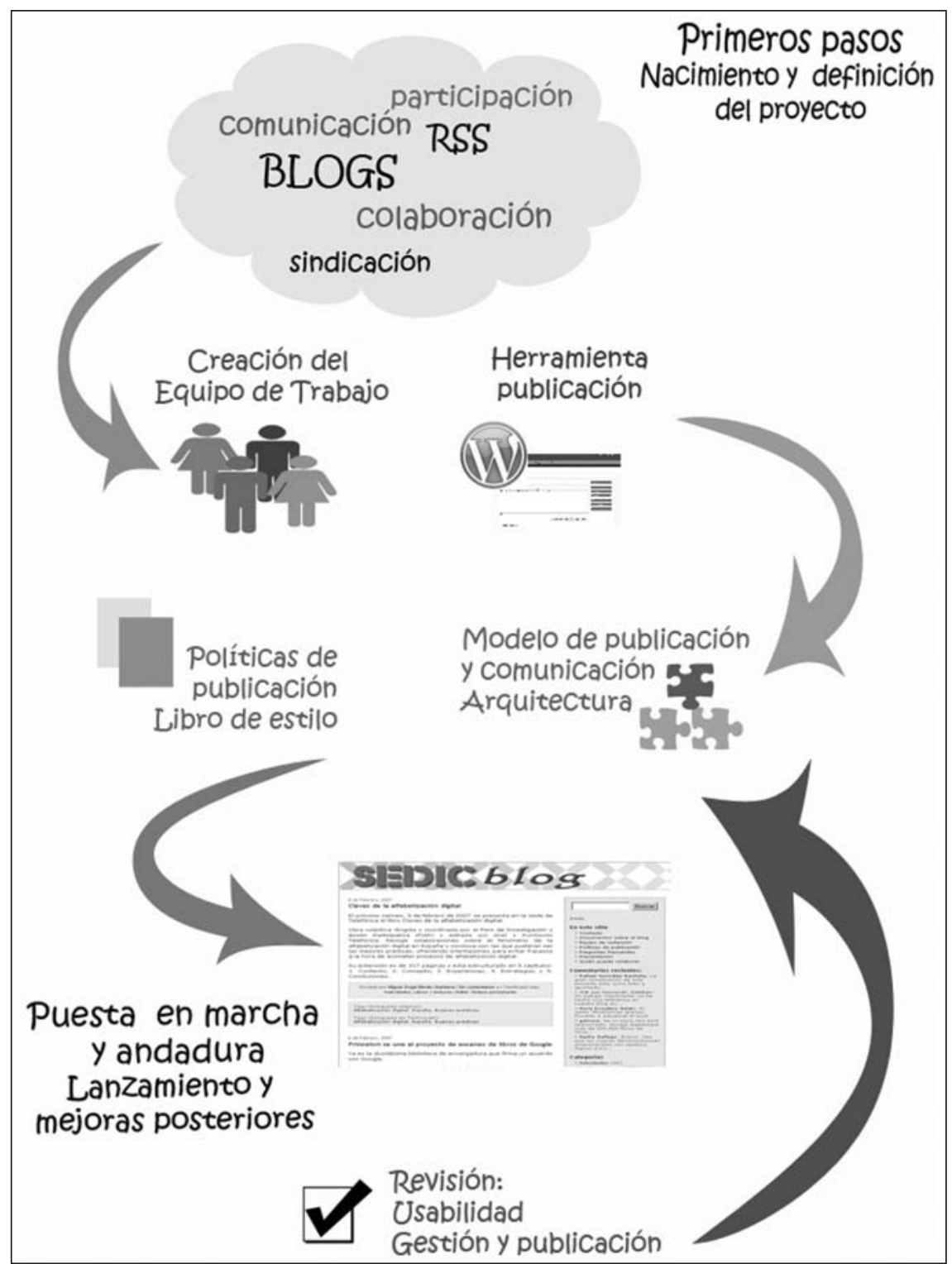

- El resultado es un medio de comunicación que está funcionando de forma continuada y está potenciando la visibilidad de la asociación. Sin embargo, el nivel de participación es aún muy modesto para un colectivo que supera los mil socios. Se ha pretendido aunar la exigencia de calidad en los contenidos y en su organización y presentación con la consecución de una verdadera conversación con el colectivo profesional. En este sentido, aún hay mucho terreno para progresar. El modelo sí ha demostrado ser válido para crear un canal colaborativo de comunicación y publicación de información web. Hasta ahora la asociación sólo utilizaba herramientas de transmisión unidireccional de información de carácter corporativo.

- La apuesta por la calidad puede resultar un freno para la captación de colaboraciones. Los documentos sobre estilo y calidad han recibido una buena acogida entre profesionales ya habituados a los blogs, pero han podido tener un efecto contrario para la participación al dar la falsa impresión de que hace falta pasar por una formación inicial para poder colaborar. Igualmente, se ha visto necesario ofrecer las máximas facilidades para la aportación de comentarios y dar la mayor visibilidad posible a los nuevos contenidos.
- Se sigue apreciando la necesidad de llevar a cabo acciones formativas y elaborar ayudas específicas y contextualizadas acerca del uso e implicaciones de la sindicación de contenidos.

\section{Bibliografía y referencias}

Barrón, Gustavo, et al. Errores de usabilidad en blogs, 2005. Consultado en: 17-07-06.

http://www.veaseademas.com/archivos/2005/11/ errores_de_usab.html

Butera Fajardo, María Jesús; Escudero Galán, Nuria. "Blogs y usabilidad: el caso del blog de Sedic". En: VIII Jornadas de gestión de la información. Madrid: Sedic, 2006, pp. 75-83. Consultado en: 12-12-06.

http://eprints.rclis.org/archive/00007896/

García, Juan Carlos. La usabilidad de las bitácoras como factor de éxito, 2006. Consultado en: 14-07-06.

http://usalo.es/141/la-usabilidad-de-las-bitacoras-como-factor-de-exito

Hassan Montero, Yusef; Martín Fernández, Francisco J. Guía de evaluación heurística de sitios web, 2003a. Consultado en: 17-07-06. http://www.nosolousabilidad.com/articulos/heuristica.htm

Leiva-Aguilera, Javier. "Breve aproximación a los blogs para unidades de información". En: Anuario ThinkEPI, 2007, pp. 166-168.

Leiva-Aguilera, Javier. "Blogs: una herramienta de difusión para profesionales de la información". En: $T K, 2006$, n. 18, diciembre, pp. 173 180

Leiva-Aguilera, Javier. "Blogs e issn: ¿una relación imposible?". En: El profesional de la información, 2006, v. 15, n. 4, julio-agosto, pp. 319-322.

Nielsen, Jakob. Weblog usability: the top ten design mistakes, 2005. Consultado en: 17-07-06. http://www.useit.com/alertbox/weblogs.html

Nielsen, Jakob. Usabilidad: diseño de sitios web. Madrid: Pearson Educación, 2002.

Nielsen, Jakob. Why you only need to test with 5 users, 2000. Consultado en: 17-07-06.

http://www.useit.com/alertbox/20000319.html

Nielsen, Jakob. How to conduct a heuristic evaluation, 1994. Consultado en: 17-07-06. http://www.useit.com/papers/heuristic/heuristic_evaluation.html

Virginia Becerril González, María Jesús Butera Fajardo, Nuria Escudero Galán, Julio Igualador Osoro, Elisa Prieto Castro y Luis Rodríguez Yunta.

sedic@sedic.es 